\title{
Simple Forward Finite Difference for Computing Reproduction Number of COVID-19 in Indonesia During the New Normal
}

\author{
Suryasatriya Trihandaru, Hanna Arini Parhusip, Bambang Susanto, Yohanes Sardjono \\ 1,2,3 Departemen Matematika dan Sains Data, Universitas Kristen Satya Wacana, Indonesia \\ ${ }^{4}$ BATAN Jogjakarta, Indonesia \\ suryasatriya@uksw.edu ${ }^{1}, \underline{\text { hanna.parhusip@uksw.edu }{ }^{2} \text {, bambang.susanto@uksw.edu }}$, \\ sardjono.batan@gmail.com ${ }^{4}$
}

\begin{abstract}
Article History:

Received : 29-11-2020

Revised : 17-03-2021

Accepted : 20-03-2021

Online : :16-04-2021

Keywords:

COVID-19;

finite difference;

reproduction number;

time dependent.

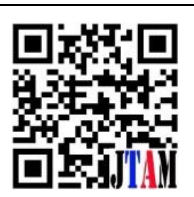

The research purpose shown in this article is describing the time dependent reproduction number of coronavirus called by COVID-19 in the new normal period for 3 types areas, i.e. small, medium and global areas by considering the number of people in these areas. It is known that in early June 2020, Indonesia has claimed to open activities during the pandemic with the new normal system. Though the number of COVID-19 cases is still increasing in almost infected areas, normal activities are coming back with healty care protocols where public areas are opened as usual with certain restrictions. In order to have observations of spreading impact of COVID-19, the basic reproduction number (Ro) i.e. the reproduction number (Ro) is the ratio between 2 parameters of SIR model where SIR stands for Susceptible individuals, Infected individuals, and Recovered individuals respectively. The reproduction numbers are computed as discrete values depending on time. The used research method is finite difference scheme for computing rate of change parameters in SIR models based on the COVID-19 cases in Indonesia (global area), Jakarta (medium area) and Salatiga (small area) by considering the number of people in these areas respectively. The simple forward finite difference is employed to the SIR model to have time dependent of parameters. The second approach is using the governing linear system to obtain the values of parameter daily. These parameters are computed for each day such that the values of Ro are obtained as function of time. The research result shows that 3 types areas give the same profiles of parameters that the rate of changes of reproduction numbers are decreasing with respect to time. This concludes that the reproduction numbers are most likely decreasing.
\end{abstract}

d.i) Crossref

https://doi.org/10.31764/jtam.v5i1.3468

\section{(c) (i) (2)}

This is an open access article under the CC-BY-SA license

\section{A. INTRODUCTION}

The infection of coronavirus disease 2019 (COVID-19) has led to pandemic with the SARSCoV-2 virus since December 2019 in Wuhan ,China and expanded globally in at least 25 countries in June 2020 (Wu et al., 2020). The current state the disease and its pathology have been examined such as estimation of total infected, and projected numbers of severe pediatric cases in 2020 (Pambuccian, 2020). Many countries have developed their efforts to handle the impact of pandemic. The group of Corona-Tracker in China for instance, utilizes outbreak data analysis and prediction using SEIR modelling to forecast COVID-19 outbreak within and outside 
of China based on daily observation (Amira et al., 2020). Multiple Linear Regression techniques are applied to the data set in India to visualize the trend of the affected cases leading to 52,290 prediction active cases after 15th August 2020 (Rath et al., 2020)(Dilip Kumar et al., 2020). The spreading of COVID-19 in the world and Indonesia in the early 1 month data from Indonesia has been used to predict the next 2 weeks in April 2020 using Support Vector Regressian (Parhusip, 2020). The result has shown that the prediction should be improved. Among these literatures, the reproduction number has been dominantly taken into account due to its used for providing transmissibility of COVID-19 in society but its formulation is still considerable complex which needs to be studied further. Therefore this article provides its formulation and its computation based on the given data of COVID-19 cases in Indonesia during the new-normal period is started to establish (since 1-st June 2020).

The basic reproduction number, Ro, indicates as the threshold parameters to predict ability of an infection to spread (Paul L et al., 2019). Therefore, the study of Ro is fundamental for learning epidemiology due to pathogen dynamic, to estimate transmission parameters during an outbreak and give a standardized of reproduction number by developing a toolbox package to do computation (Obadia et al., 2012). Based on the reproduction number computation, any activity that can increase infection was prohibited. For example, the trend of COVID-19 in the mainland of China was recognized (Kucharski et al., 2020) (Prem et al., 2020). Since mobility and population migration may impact seriously to its spreading, the lockdown policy has been taken into account to be the effective way. The lockdown of Hubei province in China for instance has significantly reduced the basic reproduction number of COVID-19. Using the SEIR model (Susceptible, Exposed, Infected, Recovered) to the spread of COVID-19 within a region in South East Asia, the R0 was very high as seen by the steep increase of people got infected and admitted to the hospitals (Putra \& Abidin, 2020) with the maximum number of cases was 2500 cases. In case of Europe, the real-time reproduction number were recorded by fitting the existing epidemic curve. It was observed that the maximum reproduction number was found in Spain and transmissibility in France (Yuan et al., 2020). Italy is also a strong contagion rate and the deterministic SEIR model was used to simulation the infected population and the number of causalities resulting 3.0 value of Ro initially and $0.57 \%$, the infection fatality rate (IFR) (Carcione et al., 2020) .Futhermore, the model was updated by the SEIR model computed with swarm intelegence(Godio et al., 2020). However, SIR model (S: suspected, I:infected, $\mathrm{R}$ :Removed/Recovered) is considerible simpler than the SEIR model as stated in the study COVID-19 cases in Morocco (Ifguis et al., 2020). Therefore, this article chooses SIR model for further discussion.

In order to use SIR model, data are required. The authors here have collected data of Suspected, Infected and Recovered individuals from 3 selected areas and cases, namely Salatiga, Jakarta are the first and second cases and from all towns in Indonesia are sum up as the third study case. This article shows the results of computations carried out from these 3 types areas for the COVID-19 cases, especially in determining the parameters in the SIR model. As it is known, the basic reproduction number parameter Ro provides information on how fast the COVID-19 virus is transmitted. We can also find out how much Ro is in various countries and there are several studies related to Ro for COVID-19 (Gray et al., 2009) (Obadia et al., 2012) where the SIR model is stochastically used. However, the used complexity cannot provide an explanation for the rate of reproduction with respect to time (Paul L et al., 2019). For this reason, this article will compute specifically the parameters of the SIR model, namely beta $(\beta)$ and gamma $(\gamma)$ so that the Ro $=\beta / \gamma$ parameter can be obtained. However, the following explanation will show how the parameters are time dependent so that the values of $S(t), I(t)$ and $R(t)$ are obtained from the given data. Unlike the model of system differential equations in general, the parameters are input to get $S(t), I(t)$ and $R(t)$ where the initial values of the three 
variables are known. The SIR models in several communities have been demonstrated (Cooper et al., 2020) (Dilip Kumar et al., 2020) where the parameters are known. In this article, we formulate the parameters in SIR model to obtain the time dependent of the basic reproduction number (Ro).

\section{B. METHODS}

There are several methods by many authors to determine the basic reproduction number due to its complexity. In Shahroud in Northeastern Iran for instance, it was assumed that the daily infectiousness follows Possion distribution leading to 2.7 with $95 \%$ interval confidence. It was found as the maximum-likelihood value of Ro in the eartly 14 days, and decreased to 1.13 after 42 days. The authors reported that by the end of May 2020, the main daily cases was about $2.9 \pm 2.0$ with 87 (48-136) new cases which is still considerable high (Khosravi et al., 2020). As stated above, the paper here uses the basic numerical method to find the first formulation of time dependent reproduction number.

\section{Forward finite difference scheme of SIR model}

Numerical method is one of the used methods in this paper to get the basic reproduction number. Forward finite difference particularly is implemented to the SIR model in this article. Although this method has been used in more complete considerable variables in the modified SIR model in Wuhan case (Ud Din et al., 2020), the paper here addresses simpler SIR model to investigate the first knowledge of parameters behaviour due to time. In the following discussion, the SIR model is used in the form:

$$
\begin{gathered}
\frac{d S}{d t}=-\frac{\beta I S}{N}, \\
\frac{d I}{d t}=\frac{\beta I S}{N}-\gamma I, \\
\frac{d R}{d t}=\gamma I .
\end{gathered}
$$

The symbols $S, I, R$ are described in the following statements. $S=$ Susceptible (vulnerability): People who do not have immunity to an infectious agent, so they may become infected if exposed; $I$ = Infectious (infectious): Residents who are currently infected and can transmit the infection to susceptible individuals; $R=$ Removed: Residents who died or recovered (immune to infection), and as a result do not affect transmission dynamics in any way when other individuals contact; To estimate $\gamma$ and $\beta$ from the data, the left hand sides of equations (1)-(3) are written discretely as.

$$
\frac{d S}{d t}=\frac{\Delta S n}{\Delta t}, \frac{d I}{d t}=\frac{\Delta I_{n}}{\Delta t}, \frac{d R}{d t}=\frac{\Delta R_{n}}{\Delta t} .
$$

By implementing forward numerical methods, one yields

$$
\begin{aligned}
& \frac{\Delta S_{n+1}}{\Delta t}=-\frac{\beta_{n+1} I_{n} S_{n}}{N}, \Delta S_{n+1}=S_{n+1}-S_{n} \\
& \frac{\Delta I_{n+1}}{\Delta t}=\frac{\beta_{n+1} I_{n} S_{n}}{N}-\gamma_{n+1} I_{n}, \Delta I_{n+1}=I_{n+1}-I_{n} \\
& \frac{\Delta R_{n+1}}{\Delta t}=\gamma_{n+1} I_{n}, \Delta R_{n+1}=R_{n+1}-R_{n} .
\end{aligned}
$$

Furthermore, the values of $\gamma$ and $\beta$ are determined by using $\Delta t=1$. In this case, so the equations become

$$
\Delta S_{n+1}=-\frac{\beta_{n+1} I_{n} S_{n}}{N} \Leftrightarrow \beta_{n+1}=-\frac{\Delta S_{n+1} N}{I_{n} S_{n}} \quad, n=1,2,3, \cdots N .
$$




$$
\Delta R_{n+1}=\gamma_{n+1} I_{n} \Leftrightarrow \gamma_{n+1}=\frac{\Delta R_{n+1}}{I_{n}} \quad, n=1,2,3, \cdots N
$$

With the second and third equations can be obtained $\gamma_{n+1}$ by subtracting the 2 -nd and 3-rd equations and taking $\Delta \mathrm{t}=1$, one yields

$$
\begin{aligned}
& \Delta I_{n+1}-\Delta R_{n+1}=\frac{\beta_{n+1} I_{n} S_{n}}{N}-2 \gamma_{n+1} I_{n} . \\
& \gamma_{n+1}=\frac{-\Delta I_{n+1}+\Delta R_{n+1}+\frac{\beta_{n+1} I_{n} S_{n}}{N}}{2 I_{n}}
\end{aligned}
$$

In this step, Excel is used to perform the computations. As mentioned above, the formulas Eq. (5) and Eq.(6) will be used to compute the descrete model of the basic reproduction number.

\section{Discretization of the SIR model into a system of linear equations}

Suppose there is a set of data containing the pairs of $\left\{x_{i}, y_{i}\right\}, i=1,2, \ldots, N$. It is assumed that the data pattern is linear meaning there are parameters $m$ and $c$ so that $f\left(x_{\mathrm{i}}\right)=m x_{\mathrm{i}}+c, i=1,2, \ldots, n$.. The values of $m$ and $c$ certainly need to be determined which are the best. This idea is developed in determining the parameters in the SIR model. By choosing $\Delta t=1$, Eq.(1)-(3) become.

$$
\begin{gathered}
-\beta S(t) I(t)=S(t+\Delta t)-S(t) \\
\beta S(t) I(t)-\gamma I(t)=I(t+\Delta t)-I(t) . \\
\gamma I(t)=R(t+\Delta t)-R(t)
\end{gathered}
$$

Numerically, one writes: $S(t+\Delta t)=S_{i+1}, S(t)=S_{i}$. as well as for other variables, then the equation (4) - (6) can be written as:

$$
\beta\left[\begin{array}{c}
-S_{i} I_{i} \\
S_{i} I_{i} \\
0
\end{array}\right]+\gamma\left[\begin{array}{c}
0 \\
-I_{i} \\
I_{i}
\end{array}\right]=\left[\begin{array}{c}
S_{i+1}-S_{i} \\
I_{i+1}-S_{i} \\
R_{i+1}-R_{i}
\end{array}\right] .
$$

We obtain a linear system, i.e.

$$
\left[\begin{array}{cc}
-S_{i} I_{i} & 0 \\
S_{i} I_{i} & -I_{i} \\
0 & I_{i}
\end{array}\right]\left[\begin{array}{l}
\beta \\
\gamma
\end{array}\right]=\left[\begin{array}{c}
S_{i+1}-S_{i} \\
I_{i+1}-S_{i} \\
R_{i+1}-R_{i}
\end{array}\right]
$$

with $A=\left[\begin{array}{cc}-S_{i} I_{i} & 0 \\ S_{i} I_{i} & -I_{i} \\ 0 & I_{i}\end{array}\right], \bar{x}=\left[\begin{array}{l}\beta \\ \gamma\end{array}\right]$ dan $b=\left[\begin{array}{c}S_{i+1}-S_{i} \\ I_{i+1}-S_{i} \\ R_{i+1}-R_{i}\end{array}\right]$.

The system of linear equations (7) is solved so that $\beta$ and $\gamma$ are obtained. It should be noted that the matrix is not a quadratic form, the values of $\beta$ and $\gamma$ will change for each data pair $i$-th and $i$ +1 -th. For this reason, the completion method is carried out in stages as shown below.

\section{Solving systems of linear equations}

Since the matrix is not a quadratic form we can follow the OLS (Ordinary Least Square) form (Hansen, n.d.) which yields the following steps:

Multiply the left and right segments by the transpose of the matrix A symbolized by $A^{\mathrm{T}}$ so that $A \bar{x}=\bar{b}$ becomes $A^{T} A \bar{x}=A^{T} \bar{b}$. By inverse to the left-hand side matrix (considered to exist) it is obtained

$$
\bar{x}=\left(A^{T} A\right)^{-1} A^{T} \bar{b}
$$

\section{How to get Reproduction number Ro?}

Note that equation (8) will be done for each data pair. Therefore we will get $\beta$ and $\gamma$ for each $\Delta \mathrm{t}$. Since Ro $=\beta / \gamma$, we get Ro for every $\Delta \mathrm{t}$. This shows that we get higher accuracy from some researchers because the values of $\beta, \gamma$ and Ro are constant, whereas in this study all three 
are time dependent. Unfortunately, the data on several days of from several agent have not been recorded properly. Moreover, the initial few rows are still 0 . Therefore, the program will make data with rows containing 0 -s are removed to avoid singularity matrix.

\section{RESULT AND DISCUSSION}

1. Parameters in SIR model and the reproduction number of COVID-19 cases in Jakarta The first considered region is Jakarta as the highest infected population in Indonesia. The region is named to be the medium region compared to the other selected regions to discuss later on (Salatiga and Indonesia). The total $(N)$ population in this period was 10,570,000 assuming no one was born or died due to diseases other than Covid-19 during this period. By implementing data to Eq. (5)-(6) , we have profile of parameters $\beta$ and $\gamma$ as shown in Fig.1.

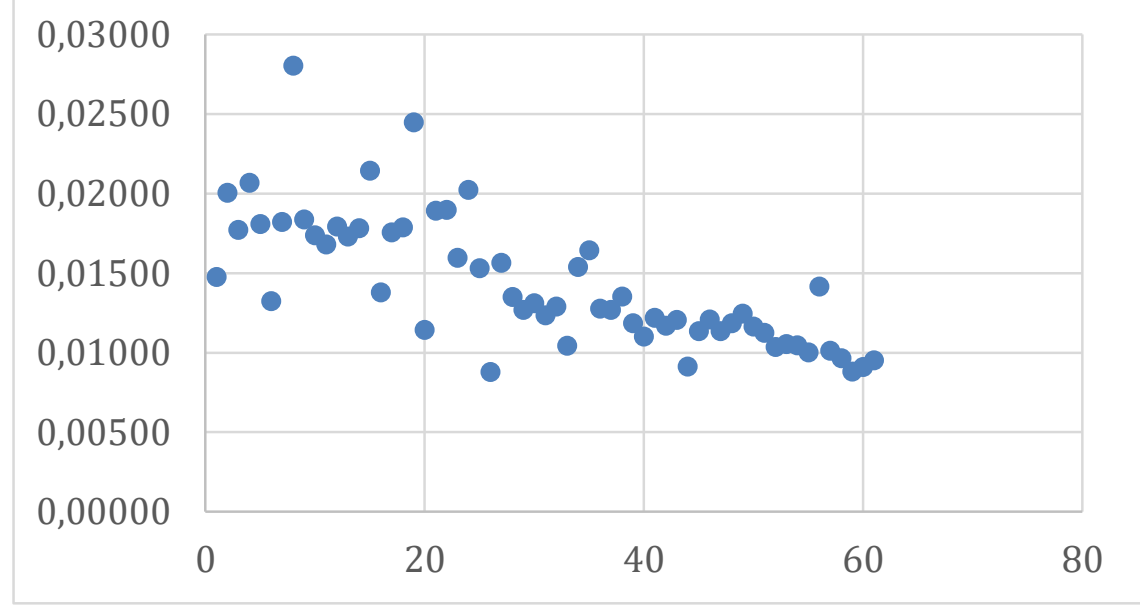

Figure 1. Value of gamma parameter (vertical) against time (horizontal) for data on COVID19 cases in Jakarta in the period 6 September-6 November 2020.

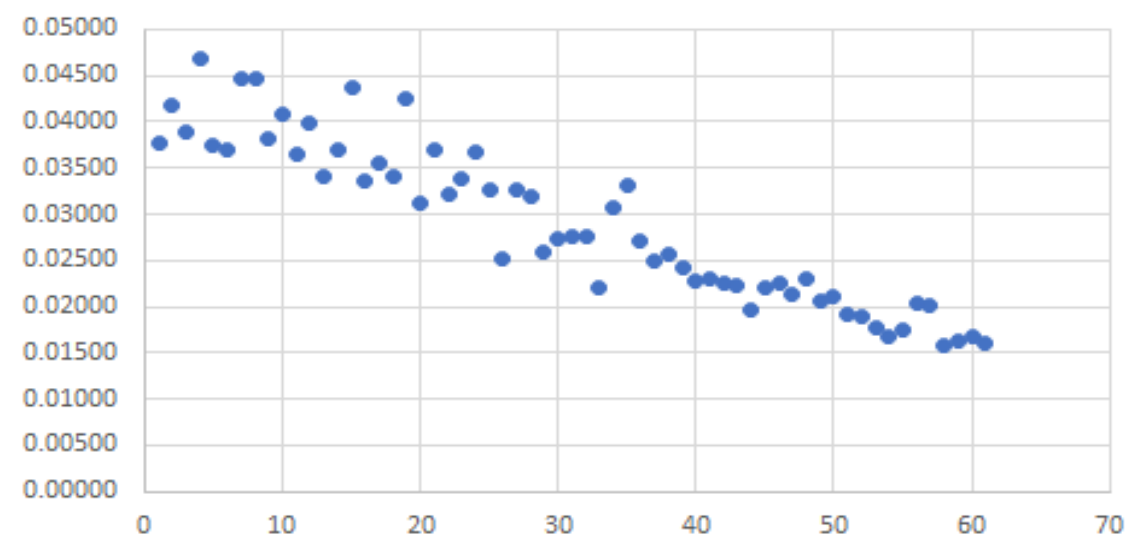

Figure 2. Beta parameter values against time for data on COVID-19 cases in Jakarta in the period 6 September-6 November 2020. 


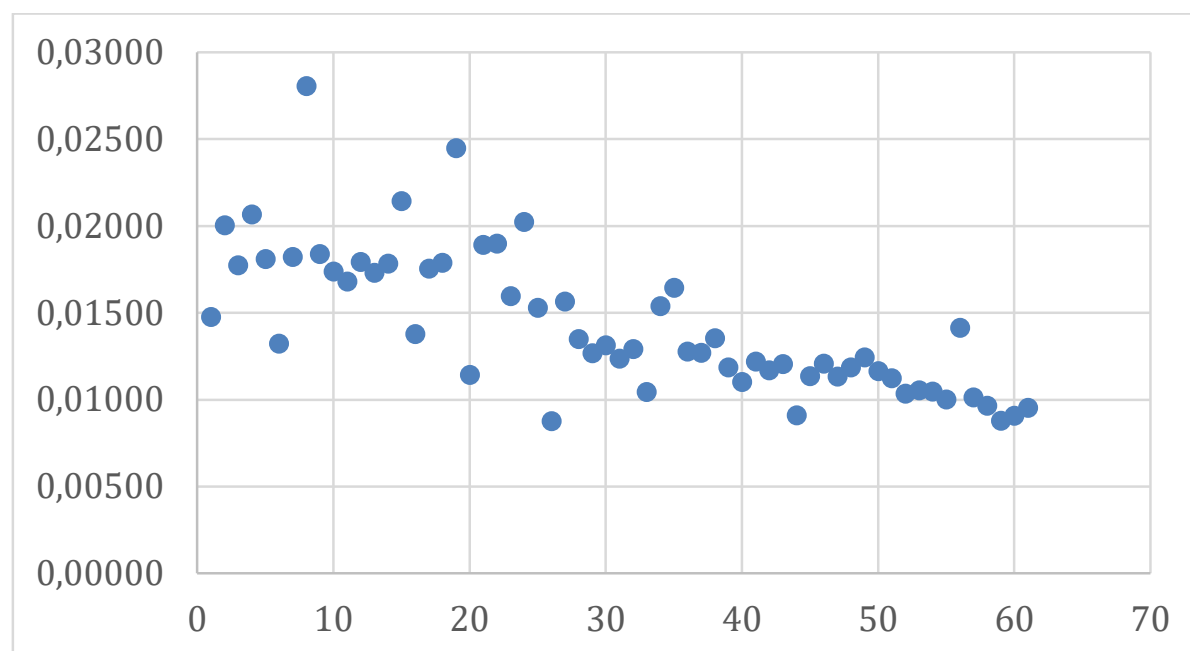

Figure 3. The value of the Ro parameter against time for data on COVID-19 cases in Jakarta in the period 6 September-6 November 2020.

From Figure 1-3, it is found that the parameter values decrease after some time of isolation so that the R0 value is getting smaller too. From the calculation of the data obtained:

The mean of $\beta=\beta^{\wedge}=0.029027394$

The average of $\gamma=\hat{\gamma}=0.014348922$

Since the values of $\beta^{\wedge}$ and $\hat{\gamma}$ have been obtained, we will look for R_0, as follows.

R_0 $=\beta^{\wedge} / \hat{\gamma}=0.029027394 / 0.014348922=2.022966802$.

Obtained R_0 $=2.022966802>1$. This value shows that one infected person has the potential to transmit the COVID-19 virus to 2-3 healthy people in Jakarta. Because of this decrease in Ro value, it is possible that Jakarta will continue to carry out new Normal activities.

\section{COVID-19 cases in Salatiga}

The used data in the COVID-19 cases data were cases on March 30-November 72020. However, some data were still empty. Hence the data were not involved so that the matrix was not singular. Therefore the data is cleaned and then the formula for the system of linear equations is used to obtain the parameter rate and basic reproduction rate. This is shown in Fig. 4. Similarly, the

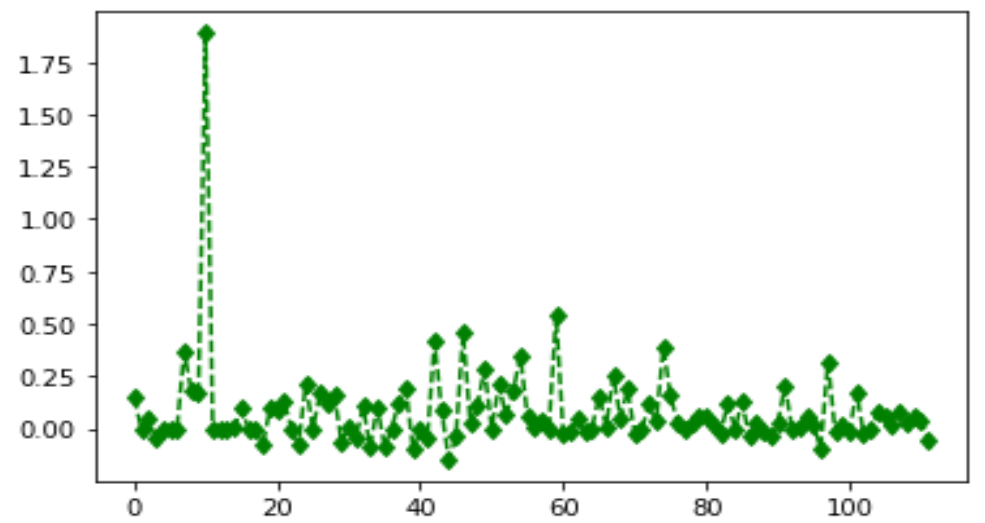

Figure 4. Beta parameter values with the SIR model containing $N=1000$,Eq.(7) against time for COVID-19 case data in Salatiga in the period 30 March - 7 November 2020. 


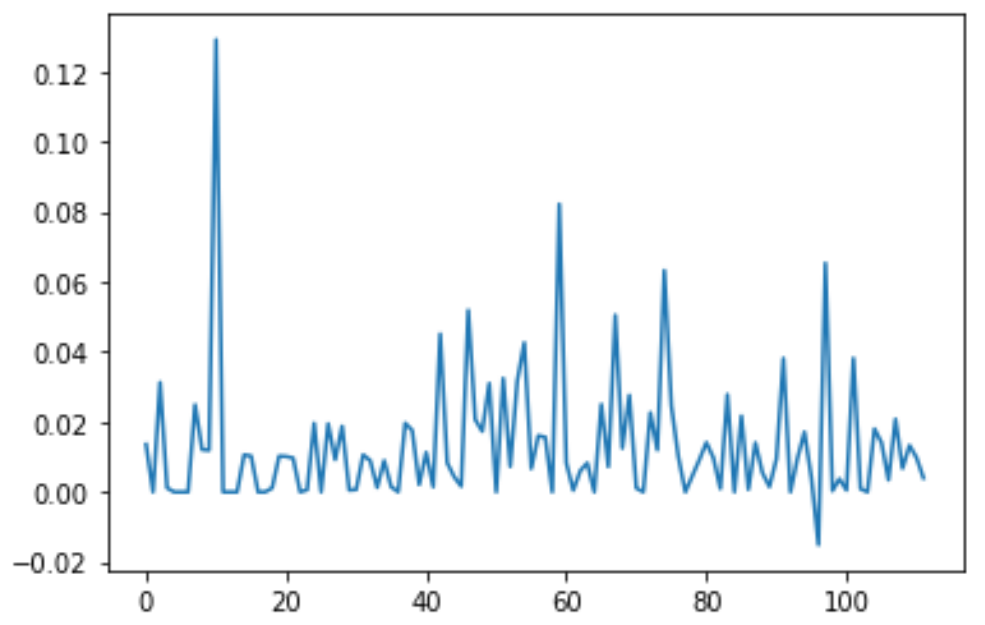

Figure 5. Gamma parameter values with the SIR model containing $N=1000$, Eq.(7) against time for data on COVID-19 cases in Salatiga in the period 30 March - 7 November 2020.

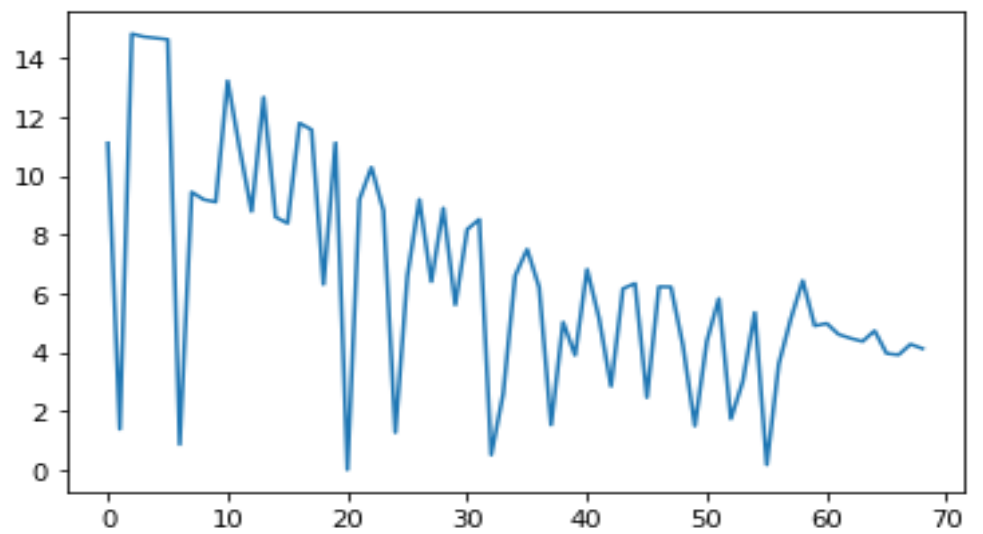

Figure 6. Value of Ro parameter with SIR model which contains $N=1000$ Eq.(7) against time for COVID-19 cases data in Salatiga in the period 30 March - 7 November 2020.

\section{COVID-19 cases in Indonesia}

The existing data are data on the total number of confirmed (positive), recovered and died cases of COVID-19 on March 2, 2020-25-September 2020. However, if there are several rows with zeo values, then the data are not used. In the same way as in the case of Salatiga, the formula for equation (7) is used. The results are shown in Figure 7-9.

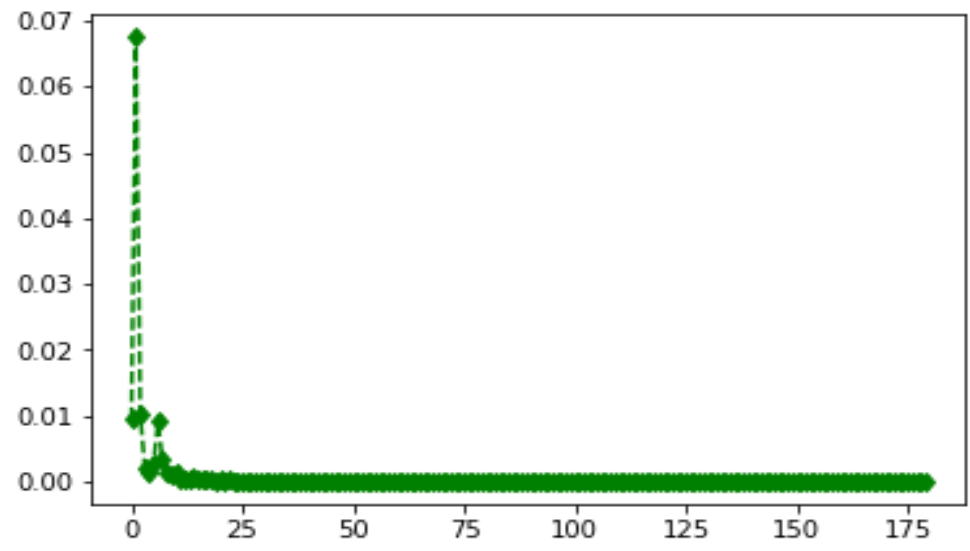

Figure 7. Beta parameter values against time for data on COVID-19 cases in Indonesia for the period 11 March - 25 September 2020. 


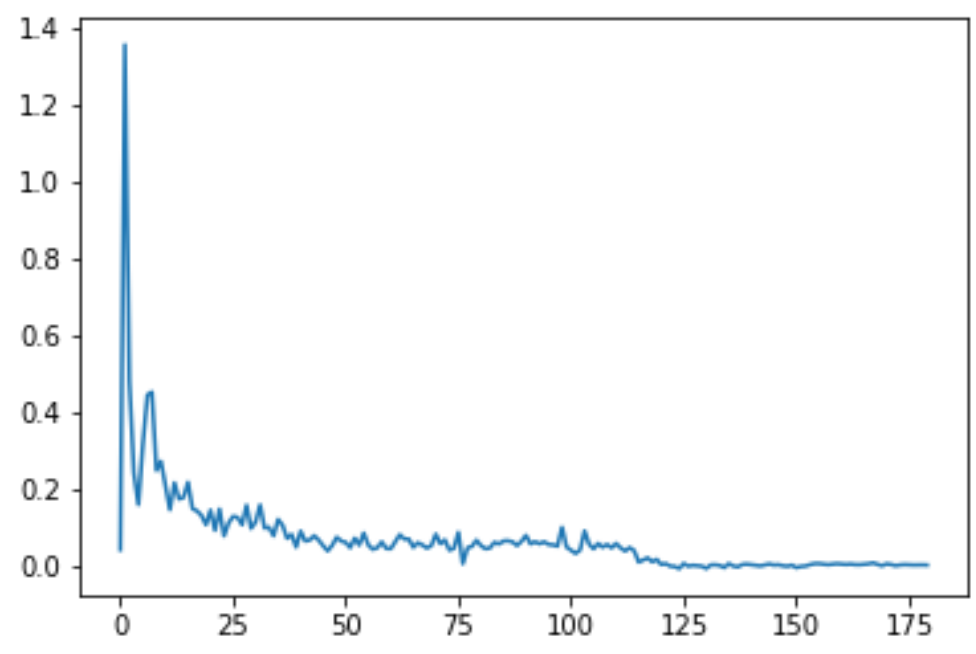

Figure 8. Gamma parameter values against time for data on COVID-19 cases in Indonesia for the period 11 March - 25 September 2020.

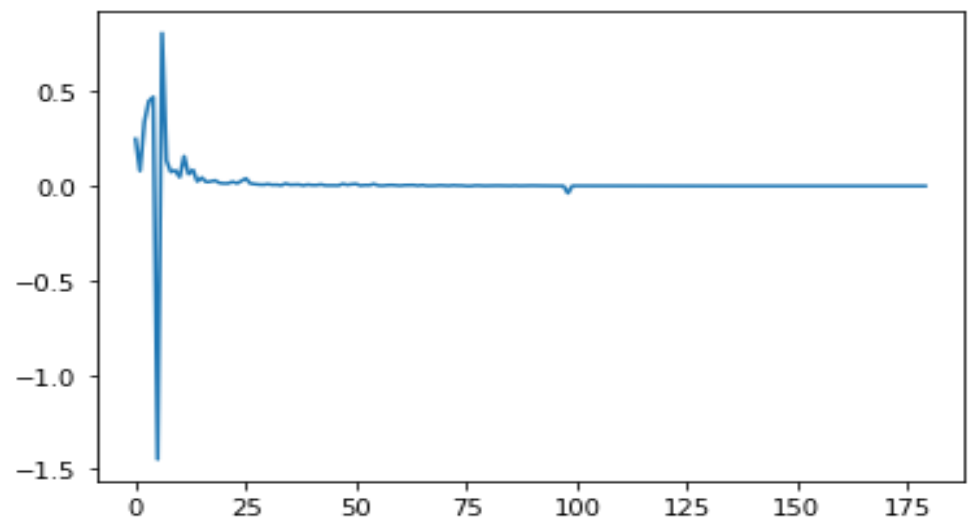

Figure 9. The value of the Ro parameter against time for data on COVID-19 cases in Indonesia for the period 11 March - 25 September 2020.

The parameter values decreased in both beta, gamma and Ro. It tends to be constant after 25 days where Ro is the value of Ro for Indonesia. Maybe it needs to be investigated more carefully whether this is true or not. In the SIR model equation (1) - (3) there is a difference with the SIR model equation (4) - (6). For this reason, the computation is carried out using models (1) - (3).

\section{Further analysis for COVID-19 cases in Indonesia}

As stated that the result of Ro is unreasonable, so the SIR model used is replaced by using models (1) - (3). This model is discretized to obtain linear system, i.e.

$$
\begin{gathered}
{\left[\begin{array}{cc}
\frac{-S_{i} I_{i}}{N} & 0 \\
\frac{S_{i} I_{i}}{N} & -I_{i} \\
0 & I_{i}
\end{array}\right]\left[\begin{array}{c}
\beta \\
\gamma
\end{array}\right]=\left[\begin{array}{c}
S_{i+1}-S_{i} \\
I_{i+1}-S_{i} \\
R_{i+1}-R_{i}
\end{array}\right]} \\
\text { therefore } A=\left[\begin{array}{cc}
\frac{-S_{i} I_{i}}{N} & 0 \\
\frac{S_{i} I_{i}}{N} & -I_{i} \\
0 & I_{i}
\end{array}\right], \bar{x}=\left[\begin{array}{l}
\beta \\
\gamma
\end{array}\right] \operatorname{dan} \vec{b}=\left[\begin{array}{c}
S_{i+1}-S_{i} \\
I_{i+1}-S_{i} \\
R_{i+1}-R_{i}
\end{array}\right] .
\end{gathered}
$$


If $N$ is taken from the population of Indonesia (200 million), then the result of Ro is also obtained unreasonable because it produces too large Ro. In some tests several $N$ values were selected. Finally, $N=1000$ was chosen because it makes the value of Ro more reasonably. It is necessary to carry out the lower and upper limits on the horizontal and vertical axes shown in Figures 10-12.

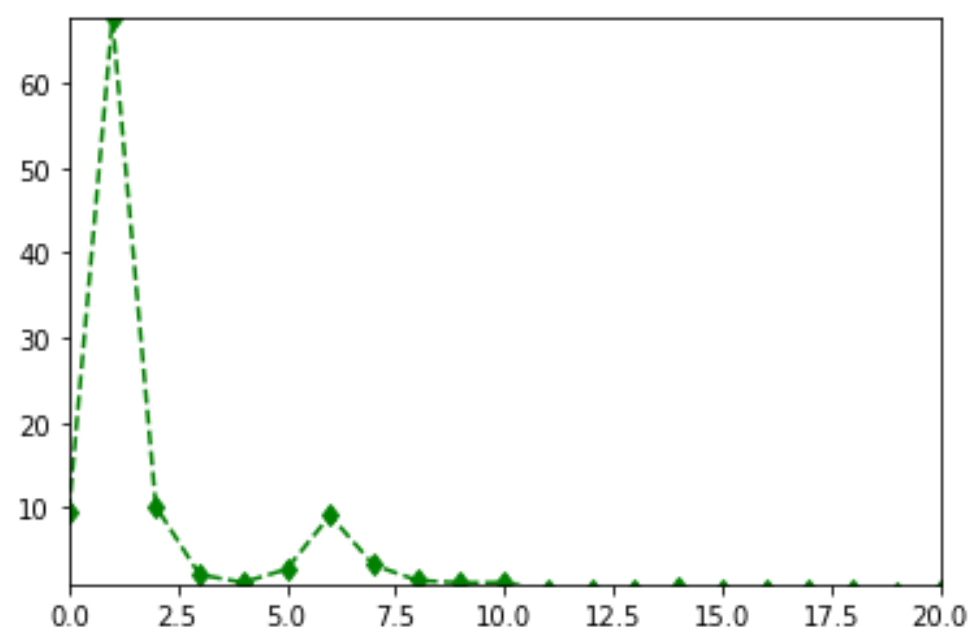

Figure 10. Beta parameter values with the SIR model containing $N=1000$,Eq. (7) , against time for data on COVID-19 cases in Indonesia for the period 11 March - 25 September 2020.

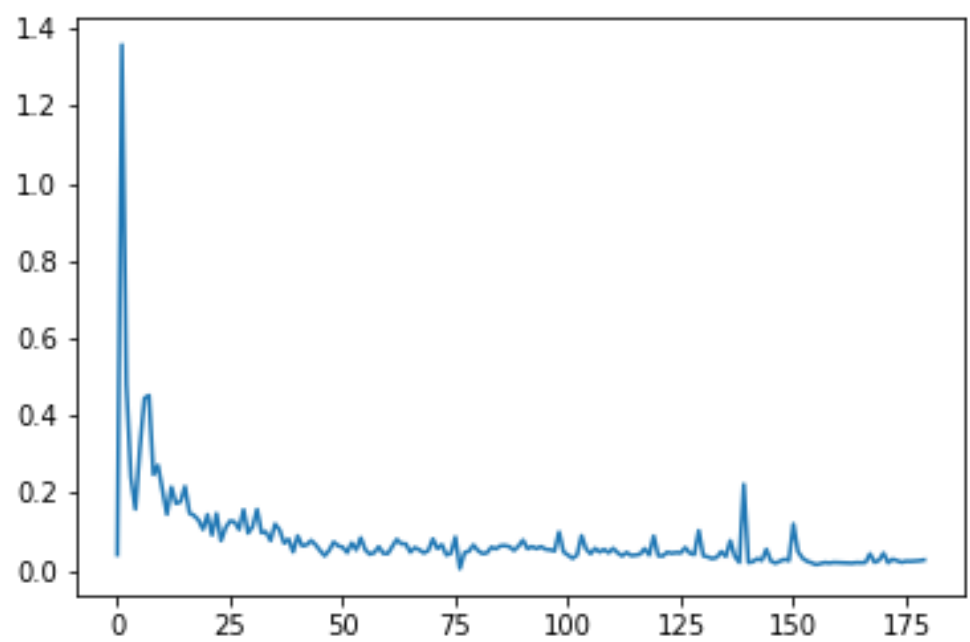

Figure 11. Value of gamma parameters with the SIR model containing N Eq.(1)-(3) against time for data on COVID-19 cases in Indonesia for the period 11 March - 25 September 2020. 


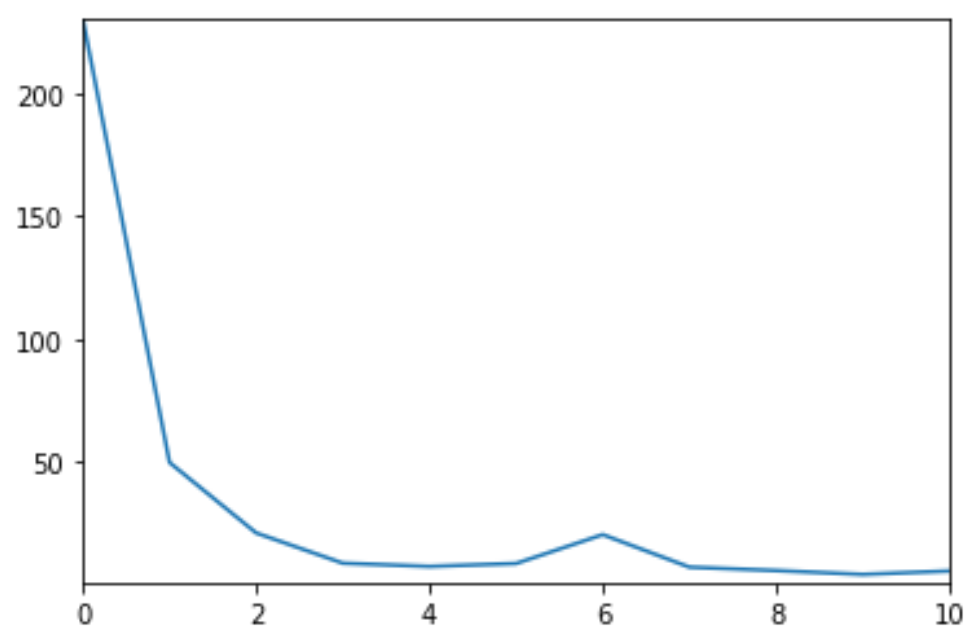

Figure 12. Value of Ro parameter with SIR model which contains N (equation (5.1) - (5.3) against time for COVID-19 case data in Indonesia for the period 11 March - 25 September 2020.

The results of Figure 7-9 show that the parameter values decrease in both the beta and gamma parameters. Likewise, the value of Ro is already a more reasonable value with the value of Ro decreasing. The results also show that the initial parameter value is unstable or oscillating so that it cannot be used as a reference in analyzing Ro. Meanwhile, the two decreasing parameters also cause the decrease in Ro as expected. So the research has shown that the value of Ro has decreased both with models Eq.(1) - Eq.(3) and with models Eq.(4) - Eq.(6).

Authors have tried to find other result from other researchers to the study of reproduction number in Indonesia. Unfortunately, the reproduction number was computed in the early transmission of COVID-19 where the reproduction number R was estimated at 6.79 and 2.47 for Jakarta-Depok and Batam respectively (Hasan et al., 2020). Therefore, the novelty of this article has shown that time dependent of reproduction number has been computed in the new normal period, which is not available in any other literature. However, the pandemic is still ongoing until this paper is written. The simulation of reproduction number in the last 4 months until March 2021 has not been studied.

\section{CONCLUSION AND SUGGESTIONS}

In this article, the rates of change parameters of the SIR model have been shown in order to obtain the basic reproduction number (called Ro) for Jakarta, Salatiga and Indonesia. These three regions were chosen to represent the location of the spread of COVID-19 cases at the scale of the most populous province (Jakarta) as a medium scale area compared to Indonesia as the spread of the COVID-19 virus globally, and Salatiga was chosen as the location for the spread of the COVID-19 virus on a local scale (small town) where population mobilization is considered quite small. Based on the three types of areas, it has been shown that the Ro value has decreased regardless of whether the Ro value is obtained. This is needed to serve as a guide for government policy makers in carrying out further policies during this pandemic. The rate of decline shows that the government's efforts have shown good results in reducing the rate of decreasing the spread of the COVID-19 virus where the Ro rate has shown a decline. Readers can also learn more about the derivation of Ro in the literatures (Hurint et al., 2017). The magnitude of the parameters is likely to be incorrect. However, the rates of parameters are very important to show the rate of change in Ro with time so that the virus spreading behavior can be known. Authors here noticed that the number of infected individuals is still growing until this month (March, 2021). Therefore, it is suggested that this article needs to be improved. 


\section{ACKNOWLEDGEMENT}

The authors would like to thank Universitas Kristen Satya Wacana who supports the research here in the periode 2020/2021 for the internal reseach project entitled CODAScI (COVID-19 Data Science Indonesia).

\section{REFERENCES}

Amira, F., Hamzah, B., Lau, C. H., Nazri, H., Ligot, D. V., Lee, G., Liang Tan, C., Khursani Bin, M., Shaib, M., Hasanah, U., Zaidon, B., Abdullah, A. B., Chung, M. H., Ong, C. H., Chew, P. Y., \& Salunga, R. E. (2020). Outbreak Data Analysis and Prediction. Bull World Health Organ. E-Pub, March. https://doi.org/10.2471/BLT.20.251561

Carcione, J. M., Santos, J. E., Bagaini, C., \& Ba, J. (2020). A Simulation of a COVID-19 Epidemic Based on a Deterministic SEIR Model. Frontiers in Public Health, 8(May). https://doi.org/10.3389/fpubh.2020.00230

Cooper, I., Mondal, A., \& Antonopoulos, C. G. (2020). A SIR model assumption for the spread of COVID19 in different communities. Chaos, Solitons and Fractals Nonlinear Science, and Nonequilibrium and Complex Phenomena, 139(January), 1-14. https://doi.org/10.1016/j.chaos.2020.110057

Dilip Kumar, B., Arati, R., Abhishek, B., \& Dulu, P. (2020). Estimating the parameters of susceptibleinfected-recovered model of COVID-19 cases in India during lockdown periods. Chaos Solitons Fractals., 2020(140). https://doi.org/10.1016/j.chaos.2020.110154

Godio, A., Pace, F., \& Vergnano, A. (2020). SEIR Modeling of the Italian Epidemic of SARS-CoV-2 Using Computational Swarm Intelligence. International Journal of Environmental Research and Public Health, 17(10). https://doi.org/10.3390/ijerph17103535

Gray, A., Greenhalgh, D., Hu, L., Mao, X., \& Pan, J. (2009). A Stochastic Differential Equation SIS Epidemic Model. SIAM Journal on Applied Mathematics, 31(5), 876-902. https://doi.org/https://doi.org/10.1137/10081856X

Hasan, A., Susanto, H., Kasim, M. F., Nuraini, N., Lestari, B., Triany, D., \& Widyastuti, W. (2020). Superspreading in early transmissions of COVID-19 in Indonesia. Scientific Reports, 10(1). https://doi.org/10.1038/s41598-020-79352-5

Hurint, R. U., Ndii, M. Z., \& Lobo, M. (2017). Analisis Sensitivitas Model Epidemi SEIR. Natural Science: Journal of Science and Technology, 6(1). https://doi.org/10.22487/25411969.2017.v6.i1.8076

Ifguis, O., El Ghozlani, M., Ammou, F., Moutcine, A., \& Abdellah, Z. (2020). Simulation of the Final Size of the Evolution Curve of Coronavirus Epidemic in Morocco using the SIR Model. Journal of Environmental and Public Health, 2020, 1-5. https://doi.org/https://www.hindawi.com/journals/jeph/2020/9769267/

Khosravi, A., Chaman, R., Rohani-Rasaf, M., Zare, F., Mehravaran, S., \& Emamian, M. . (2020). The basic reproduction number and prediction of the epidemic size of the novel coronavirus (COVID-19) in Shahroud, Iran. Cambridge University Press Public Health Emergency Collection. https://doi.org/10.1017/S0950268820001247

Kucharski, A. J., Russell, T. W., Diamond, C., Liu, Y., Edmunds, J., Funk, S., Eggo, R. M., Sun, F., Jit, M., Munday, J. D., Davies, N., Gimma, A., van Zandvoort, K., Gibbs, H., Hellewell, J., Jarvis, C. I., Clifford, S., Quilty, B. J., Bosse, N. I., ... Flasche, S. (2020). Early dynamics of transmission and control of COVID-19: a mathematical modelling study. The Lancet Infectious Diseases, 3099(20), 1-7. https://doi.org/10.1016/S1473-3099(20)30144-4

Obadia, T., Haneef, R., \& Boëlle, P. Y. (2012). The R0 package: A toolbox to estimate reproduction numbers for epidemic outbreaks. BMC Medical Informatics and Decision Making, 12(1). https://doi.org/10.1186/1472-6947-12-147

Pambuccian, S. E. (2020). The COVID-19 pandemic: Implications for the cytology laboratory. Journal of the American Society of Cytopathology. https://doi.org/https://doi.org/10.1016/j.jasc.2020.03.001

Parhusip, H. A. (2020). Study on COVID-19 in the World and Indonesia Using Regression Model of SVM, Bayesian Ridge and Gaussian. Jurnal Ilmiah Sains, 20(2), 49. https://doi.org/10.35799/jis.20.2.2020.28256

Paul L, D., Street, E. J., Leslie, T. F., Yang, Y. T., \& Jacobsen, K. H. (2019). Complexity of the Basic Reproduction Number (R0). EID, 25(1). https://wwwnc.cdc.gov/eid/article/25/1/17- 
1901_article

Prem, K., Liu, Y., Russell, T., Kucharski, A. J., Eggo, R. M., Davies, N., Group, C. for the M. M. of I. D. C.-19 W., Jit, M., \& Klepac, P. (2020). The effect of control strategies that reduce social mixing on outcomes of the COVID-19 epidemic in Wuhan, China. MedRxiv, 2667(20), 2020.03.09.20033050. https://doi.org/10.1101/2020.03.09.20033050

Putra, Z. A., \& Abidin, S. A. Z. (2020). Application of seir model in covid-19 and the effect of lockdown on reducing the number of active cases. In Indonesian Journal of Science and Technology (Vol. 5, Issue 2, pp. 185-192). https://doi.org/10.17509/ijost.v5i2.24432

Rath, S., Tripathy, A., \& Tripathy, A. R. (2020). Prediction of new active cases of coronavirus disease (COVID-19) pandemic using multiple linear regression model. Diabetes and Metabolic Syndrome: Clinical Research and Reviews, 14(5), 1467-1474. https://doi.org/10.1016/j.dsx.2020.07.045

Ud Din, R., Shah, K., Ahmad, I., \& Abdeljawad, T. (2020). Study of Transmission Dynamics of Novel COVID19 by Using Mathematical Model. Advances in Difference Equations, 2020(1). https://doi.org/10.1186/s13662-020-02783-x

Wu, Y.-C., Chen, Ching-Sunga, Chan, \& Yu-Jiuna. (2020). The outbreak of COVID-19 An overview. Journal of the Chinese Medical Association, 2020. https://doi.org/10.1097/JCMA.0000000000000270

Yuan, J., Li, M., Lv, G., \& Lu, Z. K. (2020). Monitoring Transmissibility and Mortality of COVID-19 in Europe. International Journal of Infectious Diseases, 95, 311-315. https://doi.org/10.1016/j.ijid.2020.03.050 